\title{
GROWTH OF ECCENTRICITY IN PLANET-DISC INTERACTIONS
}

\author{
J. Teyssandier ${ }^{1}$ and G. Ogilvie ${ }^{1}$
}

\begin{abstract}
The origin and wide distribution of eccentricities in planetary systems remains to be explained, in particular in the context of planet-disc interactions. Here we present a set of linear equations that describe the behavior of small eccentricities in a protoplanetary system consisting of a gaseous disc and a planet. Eccentricity propagates through the disc by means of pressure, and is exchanged with the planet via secular interactions. Excitation and damping of eccentricity can occur through Lindblad and corotation resonances, as well as viscosity. Three-dimensional effects allow for an eccentric mode to be trapped in the inner parts of the disc. This eccentric mode can easily grow within the disc's lifetime. An eccentric mode dominated by the planet can also grow, although less rapidly. Application to a hot Jupiter surrounded by a gaseous disc suggests that the eccentricity of the planet can grow. Finally, the linear theory is compared to hydrodynamical simulations, and a very good agreement is found.
\end{abstract}

\section{Introduction}

Extrasolar planets are observed to have a broad distribution of eccentricities. It has long been speculated that the interactions between a newly formed planet and the gaseous disc from which it emerged can have a significant impact on the orbital architectures of both the planet and the disc.

The pioneering work of Goldreich \& Tremaine (1980) in the context of satellites in planetary rings showed that Lindblad resonances excite eccentricities, while the effect of corotation resonances is (in general) to damp eccentricities. Simulations by various authors (e.g. Papaloizou et al. 2001; d'Angelo et al. 2006; Duffell \& Chiang 2015; Rosotti et al. 2016) argued for eccentricity growth, supported by the analysis of Goldreich \& Sari (2003). Numerical simulations on the subject are often limited to short integration times, which have to be carefully interpreted as

${ }^{1}$ Department of Applied Mathematics and Theoretical Physics University of Cambridge Wilberforce Road, Cambridge CB3 0WA, UK 
they often do not allow to follow the system on the relevant secular timescales, on which eccentricity is exchanged both reversibly and irreversibly between the disc and the planet. Analytical studies on the other hand focused on either the disc or the planet, without allowing for a coupling between the two.

Motivated by these limitations, we have developed a linear theory of eccentricity evolution of discs and planets in Teyssandier \& Ogilvie (2016a). It describes the propagation, excitation and damping of eccentricity. This model allows for the fast computation of precession rates and growth rates. We applied it to the simple case of a hot Jupiter orbiting in the inner (empty) cavity carved by the magnetosphere of a star, and perturbed by a disc expending outward. We predicted that both the disc and the planet can become eccentric.

The present work introduces the basis of a linear theory that account for both reversible and irreversible changes of eccentricity in the planet and the disc on secular timescales, in the form of a linear theory valid at small eccentricities. This linear theory is introduced in Section 2, and compared with numerical simulations in Section 3, and conclusions and direction for future work as exposed in Section 4

\section{Linear theory of eccentric discs}

In Teyssandier \& Ogilvie (2016a) we presented a set of linear equations that describe the evolution of a small eccentricity during disc-planet interactions, and applied this linear theory to the case of a hot Jupiter in an empty cavity. In this section we summarize some of the main results of this paper.

\subsection{Governing equations}

The propagation and growth or decay of eccentricity in a disc are the result of various physical processes. For small eccentricities and small eccentricity gradients, neighboring orbits do not intersect (Ogilvie 2001). It is possible to formulate a set of linear equations that describe the long-term (secular) evolution of the eccentricity, in a way that couples the eccentricity of the disc and that of the planet. Similarly to the secular dynamics of celestial mechanics, the equations are azimuthally-averaged, and quantities depend on the radial cylindrical polar coordinate $r$ and time $t$ only. In the simplest case, the disc is represented by a surface density $\Sigma$ which is a function of $r$ only, and a Keplerian rotation profile with angular velocity $\Omega=\left(G M_{*} / r^{3}\right)^{1 / 2}$. We also assume a locally isothermal disc and we define the sound speed $c_{\mathrm{s}}=H \Omega$, where $H$ is the disc scale-height. We denote by $M_{*}$ the mass of the star, $M_{\mathrm{p}}$ the mass of the planet, and $q_{\mathrm{p}}=M_{\mathrm{p}} / M_{*}$ the planet-to-star mass ratio. In this section we consider a planet orbiting in an empty cavity at a semi-major axis $a_{\mathrm{p}}$ such as $a_{\mathrm{p}}<r_{\text {in }}$, where $r_{\text {in }}$ is the inner edge of the disc.

Equations are formulated in terms of the complex eccentricity $E=e \mathrm{e}^{\mathrm{i} \varpi}$, which in the secular theory is a function of $r$ and $t$. Below we present a list of the various physical mechanisms relevant for the linear theory: 
- Pressure: A 2D secular linear theory of eccentric adiabatic discs was presented in Goodchild \& Ogilvie (2006), and isothermal discs were discussed in Teyssandier \& Ogilvie (2016a). In the case of a locally isothermal disc with sound speed $c_{\mathrm{s}}(r)$, the equation governing the propagation of a small eccentricity due to pressure has the form of a dispersive wave equation:

$$
\begin{aligned}
\Sigma r^{2} \Omega\left(\frac{\partial E}{\partial t}\right)_{\text {pressure }}= & \frac{\mathrm{i}}{2 r} \frac{\partial}{\partial r}\left(\Sigma c_{\mathrm{s}}^{2} r^{3} \frac{\partial E}{\partial r}\right)+\frac{\mathrm{i} r}{2} \frac{\mathrm{d}}{\mathrm{d} r}\left(\Sigma c_{\mathrm{s}}^{2}\right) E \\
& -\frac{\mathrm{i}}{2 r} \frac{\partial}{\partial r}\left(\Sigma \frac{\mathrm{d} c_{\mathrm{s}}^{2}}{\mathrm{~d} r} r^{3} E\right)+\frac{3 \mathrm{i}}{2} \Sigma \frac{\mathrm{d}}{\mathrm{d} r}\left(c_{\mathrm{s}} 2 r^{2}\right) E
\end{aligned}
$$

The last term of the right-hand side of this equation represents a 3-dimensional effect arising from the variation of the vertical gravitational force experienced by a fluid element as it goes along its elliptical orbit, as well as the vertical variation of the radial gravitational force (Ogilvie 2001; Ogilvie 2008).

- Secular gravitational effect: the secular potential of a planet on a circular orbit is represented by that of a ring whose mass is that of the planet:

$$
\Sigma r^{2} \Omega\left(\frac{\partial E}{\partial t}\right)_{\mathrm{pd}}=\mathrm{i} G M_{\mathrm{p}} \Sigma(r) K_{3 / 2}^{(1)}\left(r, a_{\mathrm{p}}\right) E,
$$

where $K_{3 / 2}^{(1)}$ is related to Laplace coefficients. If the disc is represented as a collection of eccentric rings, then this formulation is equivalent to the classical Laplace-Lagrange theory of planetary dynamics (Murray \& Dermott 1999).

- Viscosity: We follow Goodchild \& Ogilvie (2006) and adopt a simplified model of eccentricity damping with a Shakura-Sunyaev $\alpha$-parametrization:

$$
\Sigma r^{2} \Omega\left(\frac{\partial E}{\partial t}\right)_{\text {visc }}=\frac{1}{2 r} \frac{\partial}{\partial r}\left(\alpha \Sigma c_{\mathrm{s}}^{2} r^{3} \frac{\partial E}{\partial r}\right),
$$

where $\alpha$ is a dimensionless parameter. As stressed in Teyssandier \& Ogilvie (2016a), this effective bulk viscosity accounts for damping by any thermal or mechanical process, apart from resonances which are described below.

- Eccentric Lindblad resonances (ELR): They correspond to locations in the disc where the perturbing frequency in the rotating frame matches the epicyclic frequency. They lead to a local growth of eccentricity:

$$
\Sigma r^{2} \Omega\left(\frac{\partial E}{\partial t}\right)_{\mathrm{ELR}}=\frac{G M_{\mathrm{p}}^{2}}{M_{*}} \Sigma \mathscr{A}^{2} E w_{\mathrm{L}}^{-1} \Delta\left(\frac{r-r_{\mathrm{res}}}{w_{\mathrm{L}}} \pm 1\right) .
$$

Here $\Delta(x)=(2 \pi)^{-1 / 2} \exp \left(-x^{2} / 2\right)$ is a Gaussian representing the broadening of the resonant effect by pressure, shifted away from the nominal resonant radius $r=r_{\text {res }}$ by one resonance width $w_{\mathrm{L}}$. In addition $\mathscr{A}$ is a coefficient whose expression, as well as that of $w_{\mathrm{L}}$, can be found in Teyssandier \& Ogilvie (2016a). 
- Eccentric corotation resonances (ECR): They correspond to locations in the disc where the perturbing frequency in the rotating frame is zero. They can lead to either a growth or decay of eccentricity, depending on the local vortensity gradient, but the net effect is in general a damping of eccentricity. They read:

$$
\Sigma r^{2} \Omega\left(\frac{\partial E}{\partial t}\right)_{\mathrm{ECR}}= \pm \mathrm{d} \ln (\Sigma / \Omega) \ln r \frac{G M_{\mathrm{p}}^{2}}{M_{*}} \Sigma \mathscr{C}^{2} E w_{\mathrm{C}}^{-1} \Delta\left(\frac{r-r_{\mathrm{res}}}{w_{\mathrm{C}}}\right) .
$$

Again, the broadening of ECRs over a width $w_{\mathrm{C}}$ is represented by a Gaussian function $\Delta$, and $\mathscr{C}$ is a coefficient whose expression can be found in Teyssandier \& Ogilvie (2016a) and is a function of $r$.

In Teyssandier \& Ogilvie (2016a) we included the coupling with a non-zero planet eccentricity. We also exclude the self-gravity of the disc as well as short-range forces from the present analysis. In the simple model presented here, the disc precesses due to the combined effects of pressure and secular perturbations from the planet, and its eccentricity can grow exponentially due to eccentric Lindblad resonances, while eccentric corotation resonances and viscosity produce a damping.

\subsection{Solution in terms of normal modes}

In order to solve Equations (2.1) to (2.5), we seek normal modes of the form $E(r) \mathrm{e}^{\mathrm{i} \omega t}$. The precession rate of the mode is given by $\Re(\omega)$, while its growth rate is $-\Im(\omega)$. The method to solve these equations consists in dividing the disc into $N$ rings and associating an eccentricity to each of these rings, and is described in detail in Teyssandier \& Ogilvie (2016a).

In this section we adopt a simple disc model where the surface density follows a power law, $\Sigma \propto r^{-1 / 2}$, multiplied by a tapper function so that it smoothly falls to zero at each edge of the disc. The aspect-ratio $H$ is constant except at the inner edge of the disc where it falls to zero.

In Teyssandier \& Ogilvie (2016a), we presented a more general form that allows for the variations of eccentricity in the planet as well. For the sake of conciseness we do not report these equations here. Solving these equations in terms of normal modes shows that a mode dominated by the disc (i.e. a mode for which the AMD is predominantly in the disc) can grow on a timescale shorter than the disc's lifetime. We also find that a planet-dominated mode can grow, although on a longer timescale. On Figure 1 we show the growth rate of the planet-dominated and disc-dominated modes as a function of the disc mass. This calculation is based on parameters similar to those used in the simulations of Rice et al. (2008). The authors reported the growth of eccentricity in hot Jupiters due to a disc, but the mass of the disc was artificially pumped up by four orders of magnitude in order to speed up the calculation. Figure 1 clearly shows that in a realistic disc, the disc-dominated mode will grow more rapidly. If this mode happens to be shared between the planet and the disc, the planet could respond to the rapid growth of eccentricity in the disc by experiencing a growth of eccentricity as well. If the 


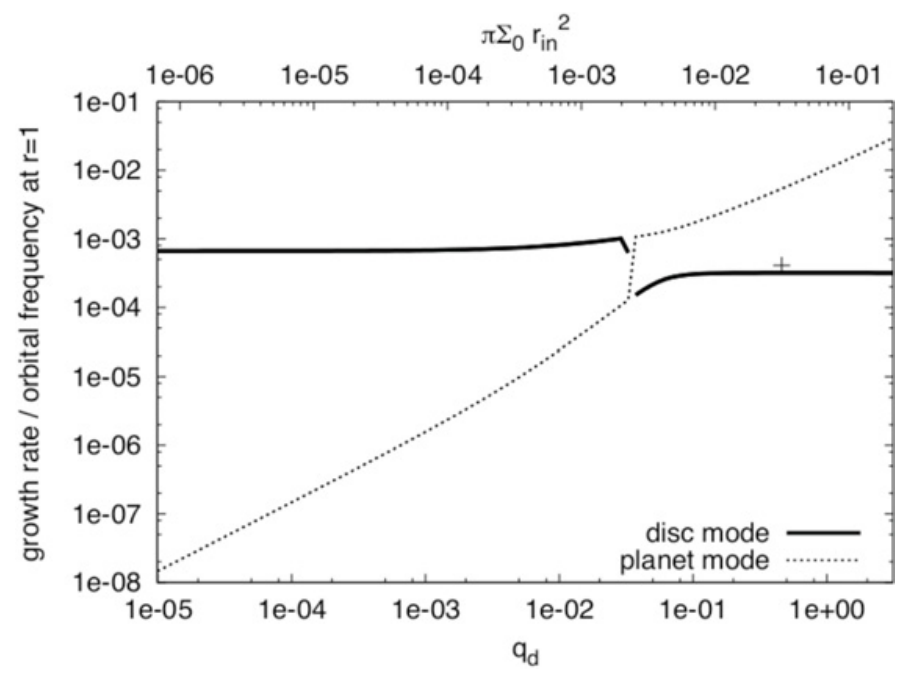

Fig. 1. Growth rate of the fastest growing disc and planet modes as a function of the disc-to-star mass $q_{\mathrm{d}}$, in a calculation similar to that of Rice et al. (2008). The cross shows the growth rate of the planet's eccentricity found by Rice et al. (2008). The upper $x$-axis shows the local mass of the disc in the vicinity of the planet. For reasonable disc masses, the disc mode is expected to grow much more rapidly than the planet mode. When the disc mass is artificially pumped up to large values $\left(q_{\mathrm{d}} \sim 1\right)$, the planet mode grows more rapidly. This illustrates why the work by Rice et al. (2008) found a growth of eccentricity of hot Jupiters due to the disc.

planet and the disc are in two largely decoupled modes, then the eccentricity of the planet could still potentially grow, although on a much longer timescale.

One important effect found in Teyssandier \& Ogilvie (2016a) was the importance of the $3 \mathrm{D}$ term in trapping a mode in the inner parts of the disc. Such trapped mode can then grow rapidly in the trapped region. An example of precession rate and growth rate of these modes in $2 \mathrm{D}$ and $3 \mathrm{D}$ are shown in Table 1. In the present work we compare our model to $2 \mathrm{D}$ simulations and therefore do not discuss further the effect of $3 \mathrm{D}$ terms.

\section{Numerical simulations}

We now wish to compare the predictions of this linear theory with direct hydrodynamical simulations. To this aim, we perform calculations using the PLUTO code (Mignone et al. 2012) on a 2D cylindrical grid, with resolution $768 \times 1422$ in radius and azimuth, and logarithmic spacing in radius. The disc is locally isothermal with a constant aspect ratio of 0.05 and a kinematic viscosity prescribed by an $\alpha$ parameter of 0.004 . The disc extends from $r=0.2$ to $r=6$. A damping zone is applied at both edges. At the inner edge, the density and velocities are 
Table 1. Precession rates and growth rates for the fastest-growing modes in $2 \mathrm{D}$ and $3 \mathrm{D}$ isothermal disc-planet systems. In 3D, the disc-dominated mode precesses progradely and grows faster than in 2D. In this calculation, the planet has a mass of $3 M_{\mathrm{J}}$, and a semi-major axis of $a_{\mathrm{p}}=0.7 r_{\text {in }}$. The disc has $H / r=0.05, \Sigma=1.3 \times 10^{-4} r^{-1 / 2}$ and $\alpha=0.004$.

\begin{tabular}{ccrc}
\hline Model & Mode & precession rate & growth rate \\
\hline 3D isothermal & Disc & $1.064 \times 10^{-3}$ & $2.985 \times 10^{-4}$ \\
& Planet & $9.524 \times 10^{-7}$ & $1.280 \times 10^{-8}$ \\
2D isothermal & Disc & $-1.533 \times 10^{-5}$ & $7.468 \times 10^{-5}$ \\
& Planet & $7.920 \times 10^{-7}$ & $1.388 \times 10^{-7}$ \\
\hline
\end{tabular}

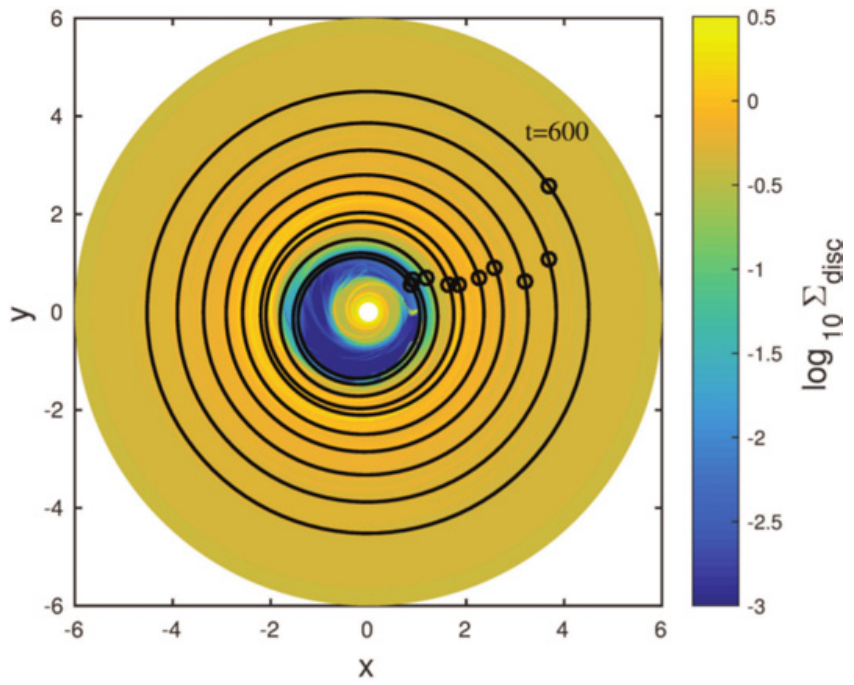

Fig. 2. Surface density of a disc with $q_{\mathrm{p}}=7 \times 10^{-3}$ after 600 orbits of the planet. Ellipses show eccentric rings fitted to the velocity field. The black circles represent the pericenter of each ellipse.

relaxed to their initial values. At the outer edge, the velocity is relaxed such as fluid elements follow circular orbits around the star-planet center of mass. The planet is kept on a fixed circular orbit at $r=1$ and we only study the growth of eccentricity in the disc. A snapshot of such set-up is shown on Figure 2 after 600 orbits of the planet. At this time an eccentric mode has grown in the disc and saturated, and the disc precesses almost rigidly.

Instead of considering the radial properties of the disc through simple azimuthal averaged quantities, we prefer to identify elliptical orbits in the disc by their semimajor axis. The eccentricity and AMD obtained from numerical simulations are 

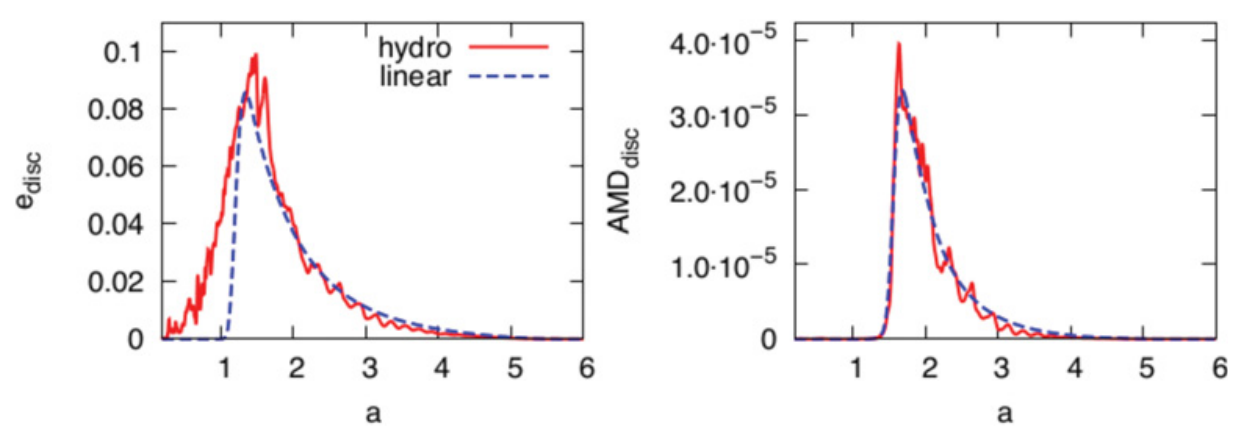

Fig. 3. Distribution of eccentricity (left) and AMD (right) in the disc as a function of semi-major axis for a $7 M_{\mathrm{J}}$ planet. The red solid line shows direct numerical simulations while the blue dashed lined shows the prediction from a linear calculation.

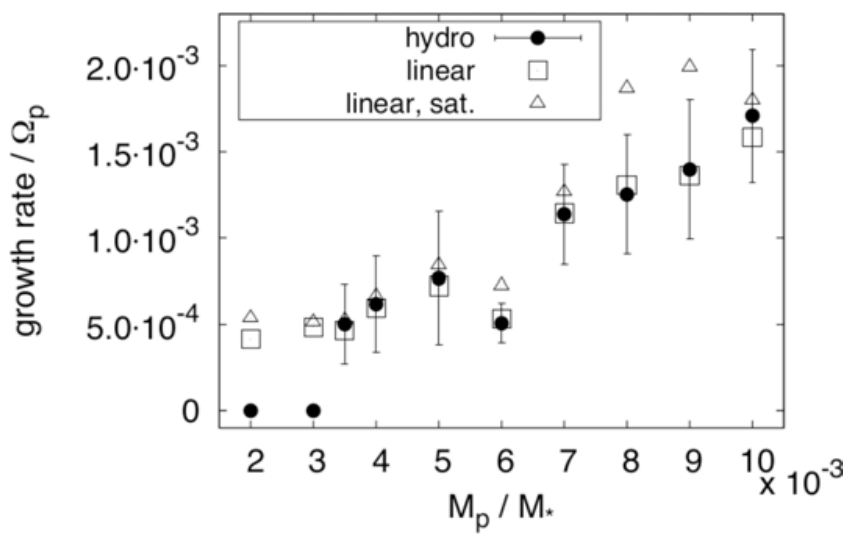

Fig. 4. Growth rate of eccentricity as a function of planet-to-star mass ratio in the disc measured from simulations (black does with error bars), and in the linear theory, both when corotation resonances are saturated (triangles) or not (squares).

compared with the results of the linear theory in Figure 3. In Figure 4 we show how the growth rate of eccentricity in the disc varies as a function of the mass of the planet. This figure also shows the growth rates computed from a linear theory, both when corotation resonances are saturated and not. These growth rates were computed taking the surface density profiles obtained in the simulations and feeding them to the linear theory. Not surprisingly, more massive planets cause the disc eccentricity to grow more rapidly. For planet-to-star mass ratios larger than 0.003 , there is an excellent agreement between the secular theory and the growth rate measured in the simulations. The fact that the disc develops some eccentricies for mass rations $\gtrsim 0.003$ is consistent with the findings of Kley \& Dirksen (2006). 


\section{Conclusion}

We have presented a linear theory of eccentricity evolution in discs and planets. This theory predicts that eccentricity can grow well within the disc lifetime. We showed that both growth rates and eccentricity distribution of protoplanetary discs perturbed by a giant planet are in excellent agreement with our linear theory. The saturation of eccentricity in a non-linear regime, as well as the possibility for the planet's eccentricity to grow remain to be studied in more details.

\section{References}

d'Angelo, G., Lubow, S.H., \& Bates, M.R., 2006, ApJ, 652, 1698

Duffell, P.C., \& Chiang, E., 2015, ArXiv e-prints 1507.08667

Goldreich, P., \& Sari, R., 2003, ApJ, 585, 1024

Goldreich, P., \& Tremaine, S., 1980, ApJ, 241, 425

Goodchild, S., \& Ogilvie, G., 2006, MNRAS, 368, 1123

Kley, W., \& Dirksen, G., 2006, A\&A, 447, 369

Mignone, A., Zanni, C., Tzeferacos, P., van Straalen, B., Colella, P., Bodo, G., 2012, ApJS, 198, 7

Murray, C.D., Dermott, S.F., 1999, Sol. Sys. Dyn., Cambridge University Press

Ogilvie, G.I., 2001, MNRAS, 325, 231

Ogilvie, G.I., 2008, MNRAS, 388, 1372

Papaloizou, J.C.B., Nelson, R.P., \& Masset, F.S., 2001, A\&A, 366, 263

Rice, W.K.M., Armitage, P.J., \& Hogg, D.F., 2008, MNRAS, 384, 1242

Rosotti, G.P., Booth, R.A., Clarke, C.J., Teyssandier, J., Facchini, S., Mustill, A.J., 2016, preprint arXiv:1609.02917

Teyssandier, J., \& Ogilvie, G.I., 2016, MNRAS, 458, 3221 